\title{
Bestimmung der Exposition der Bevölkerung in der Umgebung von GSM und UMTS Basisstationen
}

\author{
C. Bornkessel ${ }^{1}$, M. Schubert ${ }^{1}$, M. Wuschek ${ }^{2}$, and P. Schmidt ${ }^{3}$ \\ ${ }^{1}$ IMST GmbH, Kamp-Lintfort, Deutschland \\ ${ }^{2}$ Fachhochschule Deggendorf, Deggendorf, Deutschland \\ ${ }^{3}$ EM-Institut GmbH, Regensburg, Deutschland
}

\begin{abstract}
Zusammenfassung. Vorliegender Beitrag fasst die Ergebnisse von zwei Projekten aus dem Deutschen Mobilfunk Forschungsprogramm (DMF) zusammen, die vom Bundesamt für Strahlenschutz und dem Ministerium für Umwelt, Naturschutz und Reaktorsicherheit (BMU) gefördert wurden. Sie beschäftigen sich mit der Entwicklung von Messund Berechnungsverfahren zur Bestimmung der Exposition der Bevölkerung in der Umgebung von GSM- und UMTSBasisstationen sowie einer Analyse der elektromagnetischen Feldverteilung in verschiedenen Szenarien im Umfeld derartiger Sendeanlagen.
\end{abstract}

\section{Einleitung}

Zur Bestimmung der Exposition der Bevölkerung in der Umgebung von GSM- und UMTS-Basisstationen werden zunächst die örtlichen und zeitlichen Variationen der elektromagnetischen Felder untersucht. Hieraus werden geeignete Messverfahren entwickelt, die den Anforderungen der gesetzlichen Immissionsschutzverordnung entsprechen. Anschließend werden die Ergebnisse umfangreicher Messungen in der Umgebung von GSM- und UMTSBasisstationsstandorten vorgestellt. Darüber hinaus werden unterschiedliche Faktoren wie Abstand, Orientierung zur Hauptstrahlrichtung und Sichtbedingungen im Hinblick auf ihren Einfluss auf die Exposition untersucht.

Abschließend werden mehrere kommerziell verfügbare Berechnungsprogramme auf ihre Eignung bezüglich der Expositionsprognose im Umfeld von GSM- und UMTSBasisstationsstandorten überprüft. Hierfür werden die simulierten Immissionswerte mit entsprechenden Messwerten verglichen.

Correspondence to: C. Bornkessel

(bornkessel@imst.de)

\section{Messverfahren}

\section{1 Örtliche und zeitliche Feldvariationen}

Die elektromagnetischen Felder in der Umgebung von Mobilfunk Basisstationen unterliegen örtlichen und zeitlichen Schwankungen. Örtliche kleinskalige Variationen resultieren aus Fast Fading-Effekten aufgrund von Mehrwegeausbreitung, die typischerweise $10 \mathrm{~dB}$, aber auch 20 bis $30 \mathrm{~dB}$ erreichen können. Aus dieser Tatsache wird klar, dass die Ergebnisse von Messungen an einem festen Punkt nicht repräsentativ für die mittlere oder maximale Immission in einem Raum sind. Laut Anforderungen der 26. BImSchV. sind die Messungen am Einwirkungsort der größten Immission (örtliches Maximum) durchzuführen.

Darüber hinaus unterliegen die elektromagnetischen Felder in der Umgebung von Mobilfunk-Basisstationen auch klein- und großskaligen zeitlichen Variationen. Diese können anlagen- und ausbreitungswegbezogen sein. Anlagenseitige Schwankungen sind bei GSM primär bei Stationen zu finden, die über mehrere Kanäle verfügen und ab dem zweiten Kanal eine Leistungsregelung aufweisen. Diese sorgt dafür, dass entsprechend dem Verkehrsaufkommen sowohl großskalige Schwankungen im Tages- bzw. Wochenverlauf auftreten, als auch darüber liegende kleinskalige Variationen vor allem durch DTX (Discontinuous Transmission) und die verbindungsqualitätsabhängige Leistungsregelung. Daher beschreibt eine Augenblicksmessung die für eine Grenzwertaussage zwingend erforderliche Immissionssituation bei maximaler Anlagenauslastung zur unzureichend. Hier sind Techniken einzusetzen, die die gemessenen Augenblickswerte auf maximale Analagenauslastung extrapolieren.

\section{2 Örtliche Maximierung}

Mit der „Schwenkmethode“, der „Drehmethode“ und der „Punktrastermethode“ (Ryser 2003) stehen drei 
praxiserprobte, taugliche Methodiken zur Bestimmung des räumlichen Maximalwertes zur Verfügung. Alle drei Verfahren liefern unter gleichen Randbedingungen bei sorgfältiger Durchführung identische Ergebnisse (Ryser 2003). Da die Streuung der Ergebnisse aller drei Methoden vergleichbar ist und die Schwenkmethode den geringsten zeitlichen Aufwand zeigt, ist die Schwenkmethode derzeit für die hier vorliegende Aufgabenstellung am besten geeignet.

Als Antennen werden im allgemeinem bikonische oder dipolartige Antennen, isotrope Antennen (3-Achsen-Sonden) sowie logarithmisch-periodische (logper) Antennen eingesetzt. In Szenarien mit aus unterschiedlichen Richtungen einfallenden, etwa gleich starken Immissionen (z.B. Innenräume ohne direkte Sicht zur Sendeanlage) haben stärker richtende logarithmisch-periodische Antennen theoretisch Nachteile. Die schwach richtenden bikonischen Antennen bzw. Dipolantennen weisen dagegen eine teils extrem starke Beeinflussung durch den Messenden auf, die bei manchen Szenarien bis zu $10 \mathrm{~dB}$ betragen kann, bei logarithmischperiodischen Antennen aber kaum ausgeprägt ist. Diese Effekte sind bei der Aufstellung des Messunsicherheitsbudgets zu berücksichtigen.

\subsection{Zeitliche Maximierung}

Zur zeitlichen Maximierung der elektromagnetischen Felder können am effektivsten die Immissionen der mit konstanter Leistung sendenden Kontroll- oder Pilotkanäle herangezogen werden, die dann mit Hilfe der Zahl der insgesamt vorhandenen bzw. vom Regulierer genehmigten Kanäle auf die maximale Anlagenauslastung extrapoliert werden können. Hierfür werden bei GSM-Anlagen die Immissionen durch die zeitlich konstanten BCCH-Kanäle (SendeKontrollkanäle) ermittelt und mit der Zahl der maximal verfügbaren Kanäle verknüpft.

Eine korrekte Hochrechnung beim UMTS Anlagen ist möglich, in dem die Extrapolation auf Basis des kontinuierlich gesendeten Primary Common Pilot Channel (P-CPICHKanal) erfolgt, dessen Immission zuverlässig jedoch nur mit codeselektivem Messequipment erfasst werden kann.

\subsection{Zeitlich und örtlich gemittelte Messungen}

Wenn beispielsweise bei epidemiologischen Studien nicht die maximale Exposition benötigt wird, sondern zeitlich und örtliche gemittelte Immissionen, sind frequenzselektive Messungen auch bei UMTS notwendig, da die codeselektive Messung des P-CPICH nicht die zeitlichen Schwankungen durch wechselnde Verkehrsauslastung berücksichtigt. Für frequenzselektive Messungen sind die korrekten Einstellparameter des Spektrumanalysators (insbesondere die Messbandbreite RBW und der Detektortyp) sehr wichtig. Ist der Detektortyp bei GSM-Messungen nicht kritisch (Peak oder RMS, RBW sollte $200 \mathrm{kHz}$ betragen), erfordert die UMTS-Messung aufgrund des rauschähnli- chen Signalcharakters den RMS-Detektor. Messungen mit Peak-Detektor führen zu Überschätzungen des tatsächlichen Wertes von 10 bis $12 \mathrm{~dB}$. Bei Verwendung des RMSDetektors muss die Messzeit groß genug gewählt werden, da die RMS-Berechnung eine numerische Operation innerhalb des Analysators ist und hierfür eine minimale Anzahl von Samples benötigt. Für moderne Analysatoren (z.B. Rohde \& Schwarz FSP-Serie) werden Messzeiten größer als $100 \mathrm{~ms}$ benötigt, da der Messfehler ansonsten stark ansteigt. Die Auflösungsbandbreite (RBW) sollte bei UMTS $5 \mathrm{MHz}$ betragen. Messungen mit geringerer Bandbreite und anschließender Korrektur sowie Kanalleistungsmessungen sind alternativ möglich, tendieren aber zu größeren Abweichungen verglichen zu einer Referenzmessung gerade in NLOS Szenarien.

\section{Messergebnisse}

\subsection{Expositionsdaten}

In diesem Abschnitt werden Messergebnisse vorgestellt, die an folgenden Szenarien ermittelt wurden:

1. Niedrig montierte Station im ländlichen Umfeld

2. Hoch montierte Station im ländlichen Umfeld = frei stehender Mast

3. Station in einem Gewerbegebiet

4. Niedrig montierte Station im städtischen Umfeld

5. Hoch montierte Station im städtischen Umfeld

6. UHS-Station (Ultra High Site)

7. Station zur Indoor-Versorgung

8. Station zur Versorgung eines Fußballstadions

9. Station zur Versorgung einer Messehalle

10. Station zur Versorgung einer Pikozelle

11. Station auf einem Gebäudedach und Ermittlung der Immission im Gebäude

Bei der Auswahl der zu untersuchenden Anlagen wurden solche bevorzugt, bei denen neben einem UMTSSystem auch ein GSM-System installiert war. Dadurch ist es möglich, die UMTS-Expositionssituation mit der GSMExpositionssituation am selben Standort zu vergleichen. In neun Szenarien wurden sowohl GSM als auch UMTS Immissionen gemessen und in zwei Szenarien wurden nur UMTS Immissionen gemessen.

Für die Messungen wurde die Schwenkmethode mit Hochrechnung auf maximale Anlagenauslastung verwendet. Die Auswahl der Messpunkte erfolgt dabei nach systematischen Gesichtspunkten, d.h. sie weisen verschiedene Abstände, 


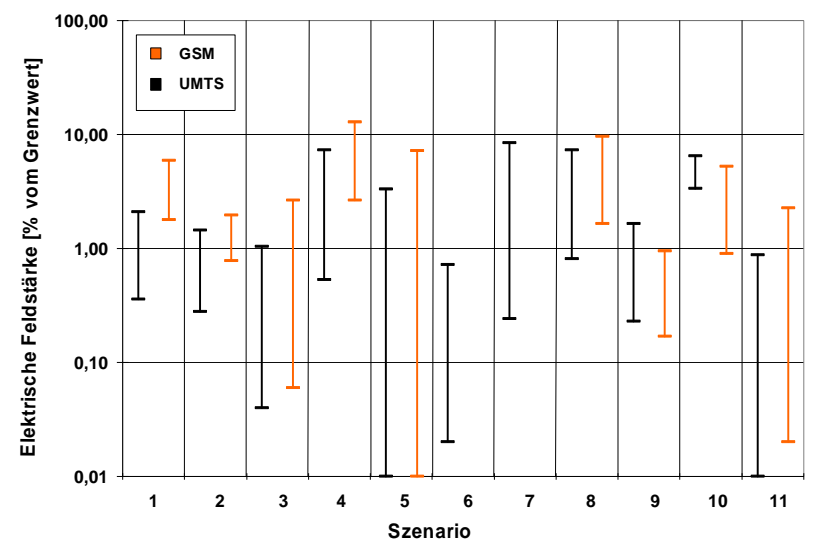

Abb. 1. Spannweite der gemessenen Immissionen an allen elf untersuchten Szenarien (Elektrische Feldstärke in Prozent der Grenzwerte der 26. BImSchV).

Orientierungen, Höhenunterschiede, Sichtverbindungen usw. zur Sendeanlage auf. Hierdurch wird in gewissen Grenzen eine Übertragbarkeit der ermittelten Immissionen auf ähnliche Szenarien ermöglicht.

Bild 1 zeigt die Spannweite der gemessenen GSM- und UMTS-Immissionen an allen elf Szenarien. Die Exposition ist dargestellt als Prozent der in der 26. BImSchV. festgelegten gesetzlichen Feldstärkegrenzwerte.

Die kleinste Immission liegt im Bereich von $0.01 \%$ der feldstärkebezogenen Grenzwerte $(0.004 \mathrm{~V} / \mathrm{m}$ bzw. $\left.0.03 \mu \mathrm{W} / \mathrm{m}^{2}\right)$ für GSM und $0.01 \% \quad(0.01 \mathrm{~V} / \mathrm{m}$ bzw. $0.1 \mu \mathrm{W} / \mathrm{m}^{2}$ ) für UMTS. Die größten gemessenen Immissionswerte betragen $12.8 \%\left(5.4 \mathrm{~V} / \mathrm{m}\right.$ bzw. $\left.78.3 \mathrm{~mW} / \mathrm{m}^{2}\right)$ für GSM und $8.4 \%\left(5.1 \mathrm{~V} / \mathrm{m}\right.$ bzw. $\left.69.3 \mathrm{~mW} / \mathrm{m}^{2}\right)$ für UMTS. Der Abstand zwischen größtem und kleinstem Immissionswert über alle Szenarien beträgt $60 \mathrm{~dB}$. Der Medianwert beträgt $0.72 \%$ bei UMTS und $1.75 \%$ bei GSM bezüglich der Grenzwerte.

Bild 1 zeigt deutlich, dass die Zellgröße und die korrespondierende Sendeleistung keine Schlussfolgerung über die resultierende Feldstärke zulassen. Der größte Immissionswert wurde in Mikrozellen-Szenarien gemessen, die zwar mit relativ kleiner Sendeleistung betrieben werden (IndoorVersorgung, Szenario 7 und 8), aber Sendeantennen an der Decke verwenden, was in kleinen Abständen zu Aufenthaltsbereichen von Personen resultiert. Ähnliche Effekte zeigten sich auch in Outdoor-Szenarien mit niedrig montierten Antennen (Szenario 4 und 10). Hoch montierte Antennen zeigten in der Regel geringere Immissionen. Dies kann auch an der UHS-Station (Szenario 6) beobachtet werden, die eine große Fläche mit großer Sendeleistung versorgt, aber die niedrigsten Expositionswerte in den hier dargestellten Messergebnissen liefert.

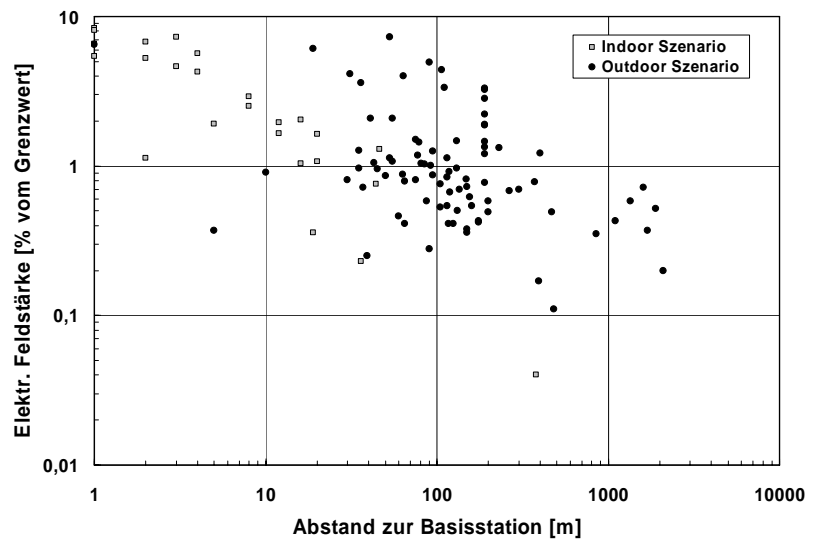

Abb. 2. UMTS Exposition als Funktion vom Abstand (Elektrische Feldstärke in Prozent der Grenzwerte der 26. BImSchV).

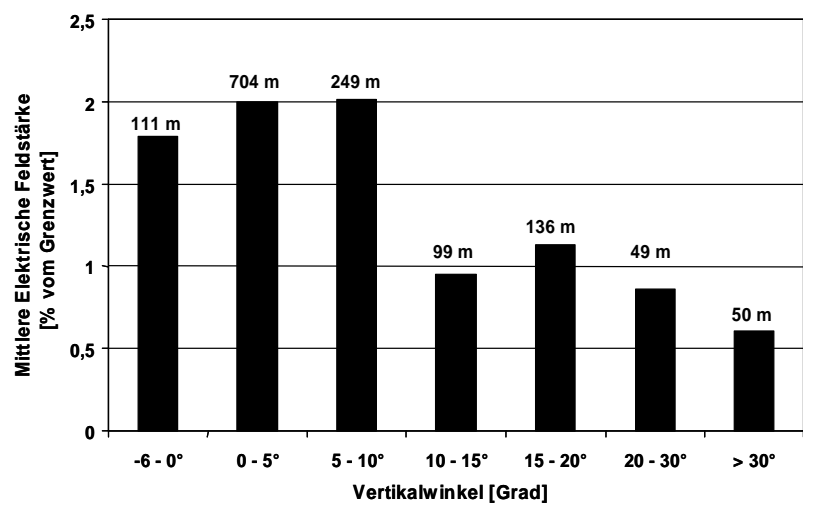

Abb. 3. UMTS Exposition als Funktion des Vertikalwinkels (Elektrische Feldstärke in Prozent der Grenzwerte der 26. BImSchV). Die Zahlen über den Balken geben den mittleren Abstand der Messpunkte zur Basisstation an.

\subsection{Einflussfaktoren auf die Exposition}

Im Folgenden werden die Einflussfaktoren für die Exposition am Beispiel der in den elf Szenarien gemessenen UMTSImmissionen untersucht. Hier hat sich herausgestellt, dass der Abstand im unmittelbaren Umfeld der Anlage (bis zu einigen hundert Metern, abhängig von Montagehöhe und Downtilt) in Outdoor-Szenarien offenbar als Maß zur Quantifizierung der entstehenden Exposition wenig geeignet ist. Grund dafür ist, dass in diesem Entfernungsbereich die Immission sehr stark durch die Nebenzipfel und Einzüge des stark bündelnden vertikalen Antennendiagramms geprägt ist und daher einen sehr unregelmäßigen Charakter aufweist. Bei Gebäuden mit Indoorversorgung ist hingegen eher eine Tendenz zur stetigen Abnahme der Exposition mit dem Abstand zu beobachten, da hier das Antennendiagramm weniger stark gebündelt ist und geringere Unterschiede im Vertikalwinkel zwischen Antenne und Expositionsort auftreten 
Tabelle 1. Untersuchte kommerziell verfügbare Software.

\begin{tabular}{llll}
\hline Software & Methode & Hersteller & Anwendung \\
\hline EFC-400 & Strahlenopt. ohne Phase & FGEU & GSM+UMTS \\
EMF-Visual & strahlenoptisch & Antennessa & GSM \\
Empire & FDTD & IMST & Allg. Untersuchungen \\
FEKO & Hybrid (z.B. MoM, UTD) & EMSS & Allg. Untersuchungen \\
FieldView & Freiraum & EM-Institut & GSM+UMTS \\
Quickplan & strahlenoptisch & TES & GSM \\
Winprop & Strahlenopt. + empirisch & AWE Communications & UMTS \\
Wireless Insite & Strahlenopt. mit Phase & Remcom & GSM+UMTS \\
\hline
\end{tabular}

(siehe Bild 2).

Ein wesentlicher Einflussfaktor für die Expositionsklassifizierung ist die Orientierung zur Hauptstrahlrichtung, die durch den Vertikalwinkel ausgedrückt werden kann, unter dem die Station vom Messpunkt aus gesehen wird. Bild 3 zeigt die UMTS Immissionen in Abhängigkeit von verschiedenen Vertikalwinkelklassen ohne Berücksichtigung von Szenario 10. Es ist deutlich zu erkennen, dass in den hier untersuchten Abstandsbereichen Punkte mit kleinen Vertikalwinkeln (zwischen $-6^{\circ}$ und $10^{\circ}$, typischer Bereich von Downtilt-Winkeln) trotz des größeren Abstandes im Mittel größere Expositionswerte aufweisen als Punkte mit größeren Vertikalwinkeln.

Auch Sichtverhältnisse üben einen deutlichen Einfluss auf die Exposition aus: Objekte wie Gebäude, aber auch Bäume und Sträucher, bzw. Wände im Indoorbereich zwischen Messpunkt und Sendeanlage haben einen dämpfenden Einfluss, der sich erheblich in den Messergebnissen widerspiegelt. Die Immissionsreduzierung ist dabei abhängig von der Dämpfungswirkung des Objektes, aber auch von der Frage, inwieweit die Signale der Basisstation auch über Reflexionen z.B. an benachbarten Gebäuden oder Wänden zum Messpunkt gelangen können.

\subsection{Vergleich von GSM und UMTS Immissionen}

Beim Vergleich der Expositionsanteile durch GSM und UMTS an identischen Standorten zeigte sich über alle Messpunkte gesehen eine deutliche Dominanz der GSMImmissionen. Grenzwertbezogen dominieren an etwa $85 \%$ der Messpunkte die durch GSM-Systeme hervorgerufenen Immissionen.

Bei der Fragestellung der Dominanz von GSM- oder UMTS-Immissionsanteilen an konkreten Messpunkten spielen mehrere Einflussfaktoren eine Rolle. Zu diesen Faktoren gehören die installierte Sendeleistung und insbesondere auch die Orientierung zur Hauptstrahlrichtung der Antennen. Die gegenüber UMTS deutlich breitere vertikale Hauptkeule von GSM 900-Antennen führt dazu, dass im Umfeld von GSM 900-Anlagen wesentlich mehr Punkte in oder nahe der vertikalen Hauptstrahlrichtung liegen als bei UMTS und somit eine höhere Immission erfahren. Letztlich sorgt auch der niedrigere Grenzwert für GSM 900-Immissionen (42 V/m bei GSM 900 im Vergleich zu $61 \mathrm{~V} / \mathrm{m}$ bei UMTS) dafür, dass bei einer grenzwertbezogenen Betrachtung oftmals die Immissionen durch GSM 900 dominieren, wohingegen sich GSM 1800 und UMTS ähnlich verhalten.

\section{Berechnungsverfahren}

\subsection{Untersuchte Software}

Neben Messverfahren wurden auch Berechnungsverfahren in den beiden hier vorgestellten Studien untersucht. Hierzu wurden speziell diejenigen kommerziell verfügbaren Programmpakete ausgewählt, die sich selbst als geeignet zur Prognose der Immissionen in der Umgebung von GSM- und UMTS-Basisstationsstandorten (außerhalb des Sicherheitsabstandes) bezeichnen. Tabelle 1 zeigt eine Übersicht der untersuchten Softwarepakete.

\subsection{Eingabeparameter}

Bezüglich der Eingabeparameter sollte die Mobilfunkantenne besonders berücksichtigt werden, denn sie ist das Element, durch das die Verteilung der Immission seitens der Basisstation bestimmt wird. In diesem Zusammenhang konnte gezeigt werden, dass das Abstrahldiagramm einer Basisstationsantenne abhängig von der Frequenz, der Montageumgebung und vom elektrischen bzw. mechanischen Downtilt ist. Daher ist es für eine Berechnung, die nicht nur eine Momentaufnahme liefern, sondern auch eine längerfristige Immissionsprognose liefern soll, sinnvoll, das Antennendiagramm so anzupassen, dass der Betriebszustand aller eingesetzten elektrischen Downtiltwinkel, der Montageumgebung und der Frequenz berücksichtigt werden kann. Dies kann z.B. durch ein synthetisiertes Antennendiagramm geschehen, das eine Umhüllende über alle Betriebszustände der Sendeantenne darstellt und für die Hauptstrahlrichtung und den Nebenzipfelbereich in Vorwärts- und Rückwärtsrichtung getrennt gebildet wird (siehe Bild 4). 
Da das Simulationsgebiet nicht nur die direkte Senderumgebung, sondern eine gesamte Versorgungszelle umfasst, muss der Geländeverlauf auf jeden Fall in der Berechnung berücksichtigt werden. Außerdem wurden Methoden vorgestellt, die es ermöglichen, auch die Vielzahl von Gebäuden in den betrachteten Softwarepaketen berücksichtigen zu können.

\subsection{Softwarebeurteilung}

Anhand eines Kataloges von verschiedenen praxisrelevanten Konfigurationen wurde ein Vergleich von berechneten Feldstärkewerten und entsprechend messtechnisch bestimmten Immissionen durchgeführt. Bild 5 zeigt die Abweichung zwischen Berechnung und Messung für Szenarien mit Sichtverbindung (LOS) für die GSM-Untersuchungen. In Bild 6 sind die Ergebnisse der Szenarien ohne Sichtverbindung (NLOS) der UMTS Untersuchungen dargestellt. Wenn das Berechnungsergebnis innerhalb eines Bereiches von $\pm 3 \mathrm{~dB}$ (typischer Bereich der Messunsicherheit) um den Messwert liegt, kann von einer guten Übereinstimmung mit dem Messergebnis gesprochen werden.

Insgesamt hat sich gezeigt, dass in Situationen mit Sicht zu Sendeantenne (LOS) alle Programme die Immission an der Mehrzahl der Vergleichsgebiete unter Berücksichtigung der Messunsicherheit gut prognostizieren. Dennoch sollten bei derartigen Konfigurationen aber folgende Aspekte berücksichtigt werden: Befinden sich z.B. Bäume in der Sichtverbindung, die in den Simulationen außer Acht gelassen wurden, überschätzen die Programme die Immission im Vergleich zur Messung. Die Größe der Überbewertung kann hierbei im Bereich von 6 bis $10 \mathrm{~dB}$ liegen. Ein Vergleich der Messresultate mit den Ergebnissen der Berechnungsmethode „Freiraum $+3 \mathrm{~dB}^{\prime \prime}$ hat gezeigt, dass auch diese Methode für Konfigurationen mit Sicht zur Anlage als geeignet angesehen werden kann. Im Gegensatz dazu ist die Methode „Freiraum $+3 \mathrm{~dB}^{\text {“ }}$ für Situationen ohne direkte Sicht zur Sendeanlage nicht geeignet, die realen Immissionen zu prognostizieren. In den betrachteten Szenarien traten hierbei Überschätzungen mit einer Schwankungsbreite von 10 bis $60 \mathrm{~dB}$ auf. Die Einbeziehung von Gebäuden in die Simulation ist dementsprechend zwingend erforderlich. Der Vergleich von Berechnung und Messung bei Szenarien ohne Sicht auf die Basisstation hat darüber hinaus gezeigt, dass die Simulationen teilweise auch noch in großer Entfernung bis zum Rand der Versorgungszelle eine gute Immissionsprognose liefern (die große Abweichung zwischen Messung und Berechnung in den Szenarien 8 und 10 resultiert aus einer Überschätzung der Immission für Bereiche direkt unterhalb der Antenne aufgrund der Auffüllung im synthetisierten Antennendiagramm).

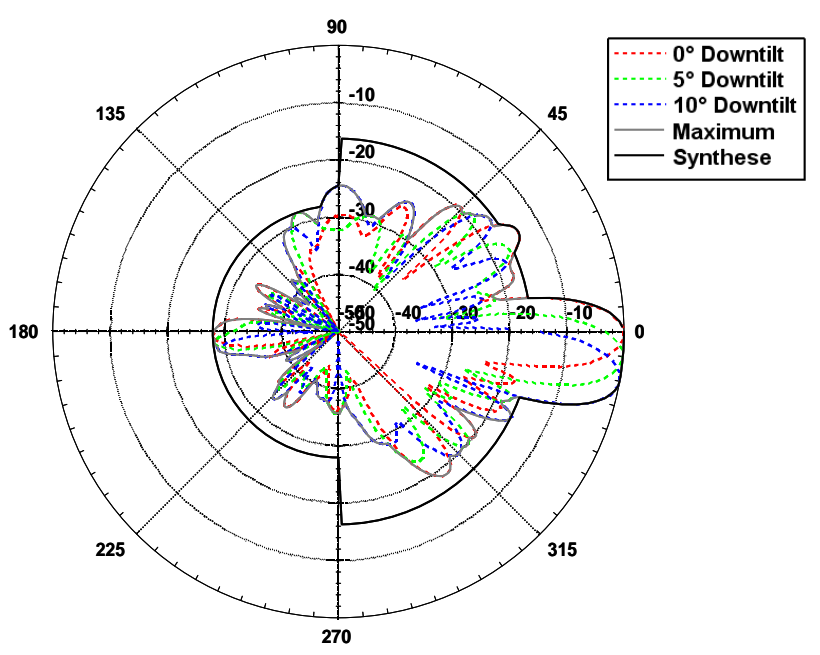

Abb. 4. Synthetisiertes Antennendiagramm.

\section{Zusammenfassung und Schlussfolgerung}

GSM- und UMTS-Expositionsmessungen der zeitlich konstanten BCCH und P-CPICH Kanäle unter Verwendung der Schwenkmethode mit anschließender Extrapolation auf maximale Anlagenauslastung resultieren in Immissionswerten, die weder von der aktuellen Anlagenauslastung noch durch Fast Fading-Effekte beeinflusst sind. Diese Vorgehensweise ist daher gut für Expositionsmessungen zur Grenzwertüberprüfung geeignet. Die neuartige codeselektive Messung bei UMTS kann mit bereits verfügbaren Messgeräten durchgeführt werden. Für zeitlich und örtlich gemittelte Messungen für epidemiologische Studien müssen frequenzselektive Verfahren und isotrope Messsonden verwendet werden, bei denen korrekte Messeinstellungen am Analysator von hoher Wichtigkeit gerade in Bezug auf UMTS sind.

Typische örtlich und zeitlich maximierte Immissionswerte in der Umgebung von GSM- und UMTS-Basisstationen liegen in der Regel unterhalb von $2 \%$ der Feldstärkegrenzwerte der 26. BImSchV. und können in seltenen Fällen mehr als $10 \%$ erreichen. Wohingegen im Nahbereich der laterale Abstand nicht als zuverlässiger Einflussfaktor auf die Exposition angesehen werden kann, haben die Orientierung zur Hauptstrahlrichtung und die Sichtbedingungen zur Antenne einen signifikanten Einfluss. In der Umgebung von Standorten, auf denen GSM- und UMTS-Systeme gemeinsam installiert sind, dominieren die GSM-Immissionen in der Mehrzahl der untersuchten Szenarien.

Mehrere kommerziell verfügbare Softwareprogramme wurden im Hinblick auf ihre Qualität der Expositionsprognose untersucht. Hierbei lieferten die meisten Programme eine gute Übereinstimmung, wenn das Antennendiagramm, das Geländeprofil und die Gebäude geeignet in den Programmen berücksichtigt wurden. Auch reine Freiraumausbreitungsprogramme lieferten in LOS-Szenarien 


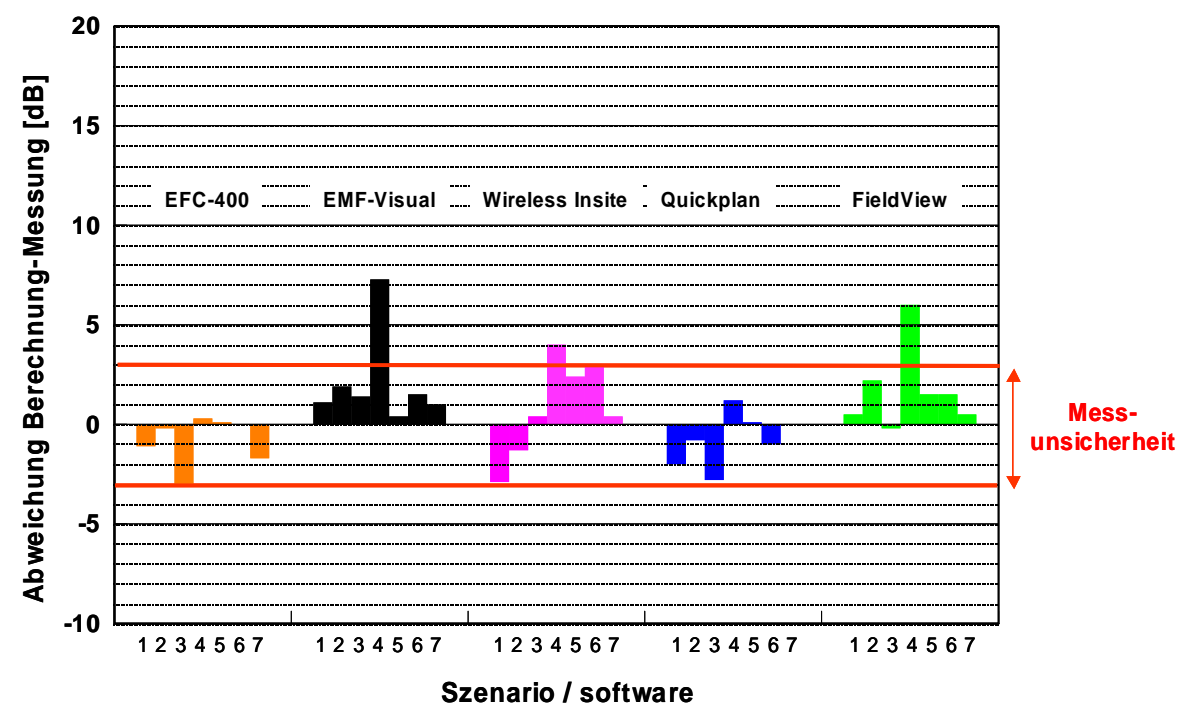

Abb. 5. Ergebnisse der Berechnungen in LOS Szenarien, GSM.

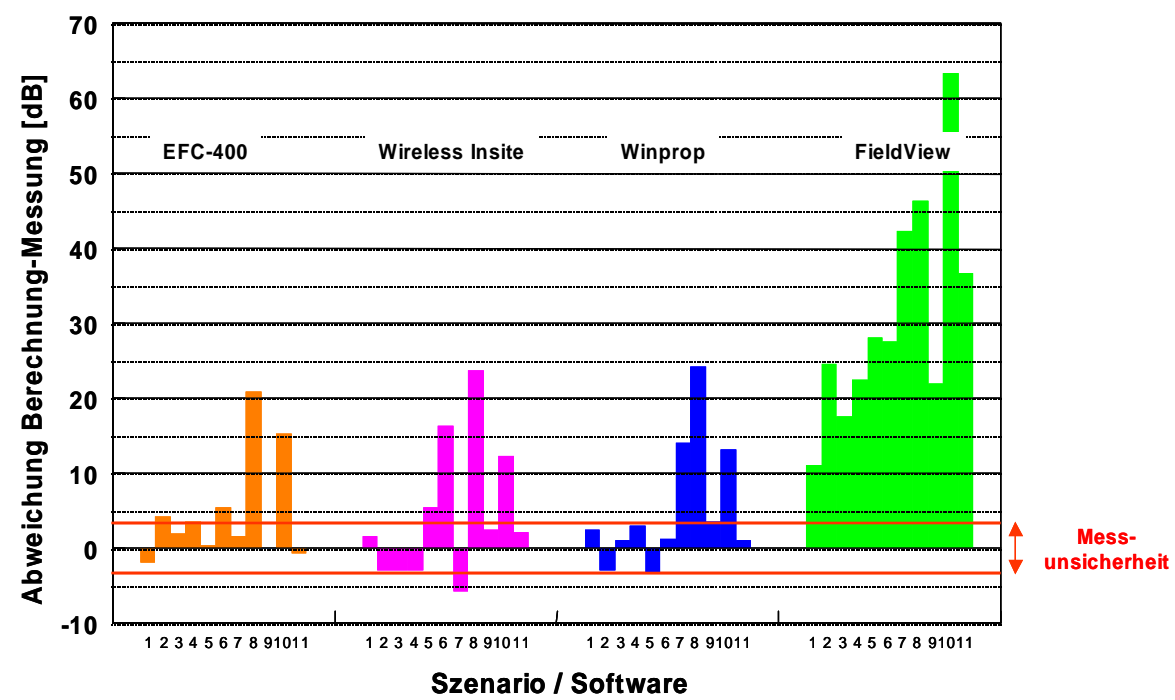

Abb. 6. Ergebnisse der Berechnungen in NLOS Szenarien, UMTS.

gute Übereinstimmungen, überschätzten die Exposition aber deutlich im Vergleich zu Messungen in NLOS-Szenarien.

Danksagung. Diese Studie wurde gefördert vom Bundesamt für Strahlenschutz und dem Ministerium für Umwelt, Naturschutz und Reaktorsicherheit (BMU) im Rahmen des Deutschen Mobilfunk Forschungsprogramms (DMF).

\section{Literatur}

26. BImSchV. Sechsundzwanzigste Verordnung zur Durchführung des Bundes-Immissionsschutzgesetzes (Verordnung über elektromagnetische Felder - 26. BImSchV), BGB1. 1996 Teil 1 Nr. 66, 20 Dezember 1996.

Ryser, H.: Measuring campaign for the assessment of the nonionising radiation near GSM base stations. 15th intl. symposium and technical exhibition on EMC Zurich, 171-174, 2003.

DMF. http://www.emf-forschungsprogramm.de/forschung, 2006. 